\title{
Relationship between rat brain and cisternal CSF tryptophan concentrations ${ }^{1}$
}

\author{
SIMON N. YOUNG, ${ }^{2}$ PIERRE ETIENNE, ${ }^{3}$ AND THEODORE L. SOURKES \\ From the Laboratory of Neurochemistry, Department of Psychiatry, \\ McGill University, Montreal, Quebec, Canada
}

SYNOPSIS Tryptophan was measured in the cisternal CSF and brains of rats. In untreated rats there was a significant but not very close correlation between the tryptophan concentration in these two compartments. Factors that change the brain tryptophan concentration such as starvation, glucose feeding, and lithium treatment affected the CSF tryptophan in the same way as the brain tryptophan. Diurnal changes were parallel for brain and CSF. When we take into account our knowledge of the disposition of tryptophan in human CSF, these data suggest that measurement of lumbar CSF tryptophan in man may be a useful approach to the study of human brain tryptophan. However, because the correlation between brain and CSF is not very close, measurements on CSF tryptophan would be more meaningful in groups of patients than in individuals.

The turnover of biogenic amines in the central nervous system (CNS) of man has been investigated by measuring the metabolites of those amines in the lumbar cerebrospinal fluid (CSF). Experiments performed primarily on experimental animals but also with man have demonstrated that the concentration of the metabolites in lumbar CSF do reflect, to a certain extent, the turnover of the parent amine in the CNS (Moir et al., 1970; Garelis et al., 1974). Thus, 5-HIAA in the CSF bears a significant relation to the brain 5-hydroxytryptamine (5-HT) content.

Recently, there has been increasing interest in the measurement of the biogenic amine precursor tryptophan in human CSF. Work on experimental animals has shown that tryptophan hydroxylase, the first enzyme on the pathway to 5-hydroxytryptamine, is not saturated with its substrate tryptophan under normal circumstances, and that changes in the brain tryptophan content will lead to changes in the rate of turn-

\footnotetext{
1 This investigation received financial support from the Medical Research Council (Canada) and the Conseil de la Recherche en Santé du Québec.

2 Address for reprints: Laboratory of Neurochemistry, Allan Memorial Institute of Psychiatry, 1033 Pine Avenue West, Montreal, Quebec, Canada H3A 1 A 1.

3 Pierre Etienne was a post-doctoral Fellow of the Conseil de la Recherche en Santé du Québec during the course of this work.

(Accepted 25 October 1975.)
}

over and concentration of 5-HT. The same may be true for man, as tryptophan administration will increase the concentration of the 5-HT metabolite 5-hydroxyindoleacetic acid (5-HIAA) in lumbar CSF. In these circumstances, after a tryptophan load, CSF tryptophan and 5-HIAA are positively and significantly correlated (Ashcroft et al., 1973b). The significant correlation implies that, in this situation, the CSF tryptophan is a good index of brain tryptophan. However, no significant correlation between tryptophan, the precursor of 5-HT, and 5-HIAA, its terminal product, has been found in the lumbar CSF of untreated patients (Ashcroft et al., 1973a; Young et al., 1974). It is of all the more interest, then, to know whether the CSF tryptophan concentration reflects the brain tryptophan content in man in normal circumstances. Therefore, we have investigated the relationship between tryptophan in these two compartments in the rat for the light that this might throw on the situation in man.

\section{METHODS}

Male Sprague-Dawley rats weighing $300 \mathrm{~g}$ were obtained from Canadian Breeding Farm and Laboratories, Ltd, St Constant, Quebec. To obtain CSF the rats were anaesthetized with pentobarbitone 


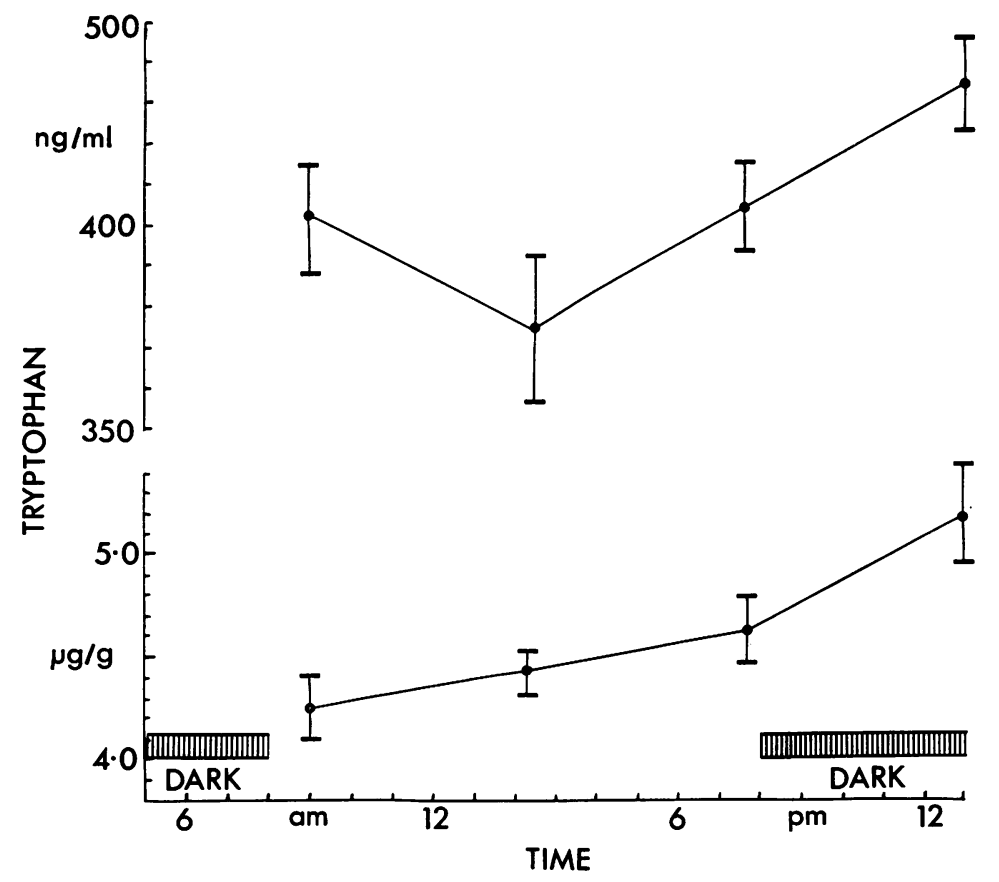

FIG. 1 The diurnal variation of rat CSF and brain tryptophan.

Rats were treated as described in the Methods section. Values in $n g / m l C S F$ (above) and in $\mu g / g$ tissue (wet weight) for brain (below) are given as mean and standard error. The number of determinations for each point is given in Table 2.

TABLE 1

EFFECT OF STARVATION AND LITHIUM ADMINISTRATION ON BRAIN AND CSF TRYPTOPHAN

\begin{tabular}{lccc}
\hline & No. of rats & Brain tryptophan & CSF tryptophan \\
\hline Fed & 7 & $4620 \pm 110$ & $420 \pm 26$ \\
Starved & 8 & $5500 \pm 193$ & $514 \pm 23$ \\
$\%$ change & & 19 & 22 \\
P & & $<0.005$ & $<0.05$ \\
Control & 7 & $5180 \pm 140$ & $400 \pm 34$ \\
Lithium & 7 & $6360 \pm 390$ & $506 \pm 38$ \\
$\%$ change & & 23 & 23 \\
P & & $<0.05$ & $\sim 0.05$ \\
\hline
\end{tabular}

Values in $\mathrm{ng} / \mathrm{g}$ tissue wet weight (brain) and $\mathrm{ng} / \mathrm{ml}$ (CSF) are given as mean $\pm S E$. The experiments were performed as described in the Methods section.

sodium (Nembutal), the atlanto-occipital membrane was exposed, and CSF taken from the cisterna magna by puncturing the membrane. The volume of CSF was determined by weighing it (assuming a specific gravity of 1.00 ) and tryptophan was determined on single samples of CSF and brain $(25 \%$ homogenate $\mathrm{w} / \mathrm{v}$ ) by the method of Denckla and Dewey (1967). Cerebrospinal fluid and brain samples were taken within five minutes of administration of pentobarbitone sodium, which was shown not to affect brain tryptophan within this time period. The
CSF volume obtained varied between 0.08 and 0.15 $\mathrm{ml}$. Samples containing blood were discarded. In the diurnal variation experiment, rats were housed in a

TABLE 2

CORRELATION BETWEEN CSF AND BRAIN TRYPTOPHAN

\begin{tabular}{lccc}
\hline Experiment & No. of rats & $r$ & $P$ \\
\hline 1 Diurnal variation & & & \\
9.00 a.m. & 6 & 0.85 & $<0.05$ \\
2.20 p.m. & 6 & 0.61 & NS \\
7.40 p.m. & 8 & 0.12 & NS \\
1.00 a.m. & 8 & 0.27 & NS \\
Whole group & 28 & 0.41 & $<0.05$ \\
2 Starvation & & & \\
Fed & 7 & 0.69 & NS \\
Starved & 8 & 0.36 & NS \\
Whole group & 15 & 0.73 & $<0.01$ \\
3ithium administration & & & \\
Saline & 7 & 0.22 & NS \\
Lithium & 7 & 0.59 & NS \\
Whole group & 14 & 0.62 & $<0.05$ \\
Glucose administration & & & \\
Control & 7 & 0.31 & NS \\
Water 1 h & 6 & 0.91 & $<0.05$ \\
Water 2 h & 6 & -0.11 & NS \\
Glucose 1 h & 6 & 0.89 & $<0.05$ \\
Glucose 2 h & 8 & -0.55 & NS \\
Whole group & 33 & 0.38 & $<0.05$ \\
\hline
\end{tabular}

The experiments were performed as described in the Methods section. NS, $\mathbf{P}>0.05$. 


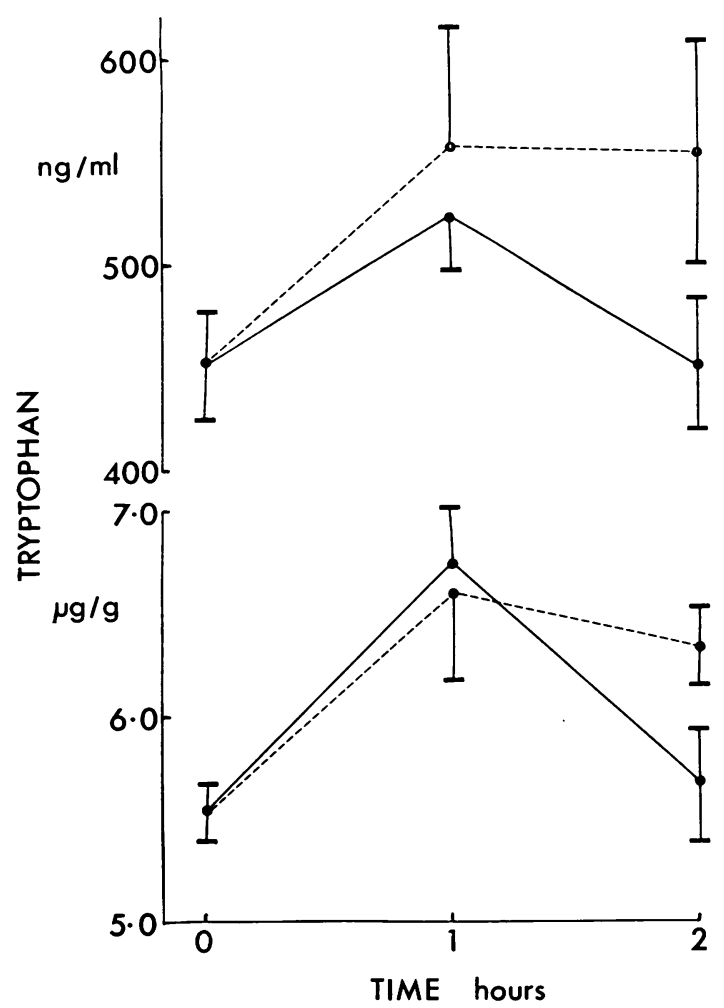

FIG. 2 The effect of oral glucose administration on rat CSF and brain tryptophan. Rats were given glucose solution (--) or water (-) as described in the Methods section. Values in $\mathrm{ng} / \mathrm{ml}$ CSF (above) and in $\mu \mathrm{g} / \mathrm{g}$ tissue (wet weight) for brain (below) are given as mean and standard error. The number of determinations for each point is given in Table 2.

constant 12 hour light-dark cycle for 10 days before the experiment. In the starvation experiment, food but not water was taken from the rats at 9.00 a.m. and the rats were killed at $9.00 \mathrm{a} . \mathrm{m}$. on the next day. In the experiment on the effect of glucose, rats were fed $2 \mathrm{ml} 50 \%(\mathrm{w} / \mathrm{v})$ glucose solution or water by stomach tube. In the experiment on lithium, rats were injected intraperitoneally with a solution of lithium carbonate $(60 \mathrm{mg} / \mathrm{kg})$ or saline twice daily for six days. The rats were killed six hours after the last injection (Perez-Cruet et al., 1971).

\section{RESULTS}

Figure 1 shows the diurnal variation of CSF brain tryptophan. The concentration of tryptophan in both is significantly higher at $1.00 \mathrm{a} . \mathrm{m}$. than at 2.20 p.m. In addition, the brain tryptophan is significantly higher at 1.00 a.m. than 8.00 a.m.

Table 1 shows the effects of starvation and lithium administration on brain and CSF tryptophan. Both treatments cause a significant increase in tryptophan in both compartments. The increase is of the same order in the two compartments.

Figure 2 shows the effect of oral administration of water or glucose solution on brain and CSF tryptophan concentrations. For both compartments the changes are of the same direction and magnitude, although for CSF, because of the great variability, none of the changes is statistically significant. Glucose, as expected, causes a rise in brain tryptophan at both one and two hours. The rise in brain tryptophan seen at one hour after water administration may have been due to the stress of handling the rats (Curzon et al., 1972).

Table 2 gives the correlations between CSF and brain tryptophan concentrations. In all cases, when the results for all the rats in a particular experiment are pooled there is a positive and significant correlation. Taking groups of rats that had received the same treatment, the variability is in the majority of situations too great for the correlation to achieve a level of significance.

\section{DISCUSSION}

Several reports have been published on factors that influence lumbar CSF tryptophan in man. Thus, intake of the amino acid is important. CSF tryptophan starts to increase two hours after a tryptophan load and reaches a maximum at eight hours (Eccleston et al., 1970). Another factor that may control CSF tryptophan is the proportion of serum tryptophan that is freethat is, not bound to albumin (Perez-Cruet et al., 1974; Young et al., 1976). Disturbed liver function affects the metabolism of the amino acid, for in hepatic cirrhosis the CSF tryptophan is greatly elevated (Young et al., 1975). Finally, mental depression is said to be associated with low tryptophan concentration in the CSF (Coppen et al., 1972), although this has not been confirmed (Ashcroft et al., 1973a). Do these results on CSF tryptophan imply that the same is 
true for the brain tryptophan? The correlation between CSF tryptophan and 5-HIAA after a tryptophan load (Ashcroft et al., 1973b) is evidence that, in these circumstances, the CSF tryptophan does reflect the brain concentration, at least within the compartment(s) where serotonin is formed and metabolized. Consistent with this is the finding that, in rats given a diet supplemented with large amounts of tryptophan, there is a correlation between the increase in the brain and in the CSF concentrations of that compound (Modigh, 1975). However, this does not necessarily imply that the small variations in brain tryptophan seen under physiological circumstances will be accompanied by similar variations in CSF tryptophan.

In the present study, we have looked at a number of factors that are known to affect brain tryptophan such as diurnal variation (Fernstrom and Wurtman, 1974), 24 hour starvation (Curzon et al., 1972), glucose administration (Madras et al., 1973), and lithium administration (Tagliamonte et al., 1971). Thus, our work has included both physiological and pharmacological factors that affect brain tryptophan. In all these situations, the changes in CSF tryptophan were comparable with those of brain tryptophan, although the CSF values showed greater variability. After glucose administration the changes in CSF tryptophan were too variable to be statistically significant. This variability may have been due in part to changes in the rate of CSF production. In cats, an increase in serum osmolarity caused by intravenous glucose infusion will lower the rate of CSF production (Hochwald et al., 1974).

Cisternal CSF was taken from the rats in this work and lumbar CSF is normally used in man. However, this is probably not of importance as tryptophan seems to have a uniform concentration in different CSF compartments in man (Young et al., 1973; Young et al., 1976). The fact that human lumbar CSF tryptophan does not start to increase until two hours after a tryptophan load (Eccleston et al., 1970) might indicate that the change in CSF tryptophan lags behind the changes in the CNS. However, as no such effect was seen in the present study, this effect may be important only for large changes brought about in tryptophan disposition by loading or by pharmacological agents. This idea is supported by the fact that CSF and free serum tryptophan show parallel variation throughout a 24 hour period (Young et al., 1976). Thus, if human CSF tryptophan is controlled by the same factors that influence rat CSF tryptophan, this study indicates that measurements of lumbar CSF tryptophan in man will provide a good index of brain tryptophan. However, because of the variability of the CSF results, measurements would have greater predictive value for groups of patients than for particular individuals.

\section{REFERENCES}

Ashcroft, G. W., Blackburn, I. M., Eccleston, D., Glen, A. I. M., Hartley, W., Kinloch, N. E., Lonergan, M., Murray, L. G., and Pullar, I. A. (1973a). Changes on recovery in the concentrations of tryptophan and the biogenic amine metabolites in the cerebrospinal fluid of patients with affective illness. Psychological Medicine, 3, 319-325.

Ashcroft, G. W, Crawford, T. B. B., Cundall, R. L., Davidson, D. L., Dobson, J., Dow, R. C., Eccleston, D., Loose, R. W., and Pullar, I. A. (1973b). 5-Hydroxytryptamine metabolism in affective illness: the effect of tryptophan administration. Psychological Medicine, 3, 326-332.

Coppen, A., Brooksbank, B. W. L., and Peet M. (1972). Tryptophan concentration in the cerebrospinal fluid of depressive patients. Lancet, 1, 1393.

Curzon, G., Joseph, M. H., and Knott, P. J. (1972). Effects of immobilization and food deprivation on rat brain tryptophan metabolism. Journal of Neurochemistry, 19, 19671974.

Denckla, W. D., and Dewey, H. K. (1967). The determination of tryptophan in plasma, liver and urine. Journal of Laboratory and Clinical Medicine, 69, 160-169.

Eccleston, D., Ashcroft, G. W., Crawford, T. B. B., Stanton, J. B., Wood, D., and McTurk, P. H. (1970). Effect of tryptophan administration on 5-HIAA in cerebrospinal fluid in man. Journal of Neurology, Neurosurgery, and Psychiatry, 33, 269-272.

Fernstrom, J. D., and Wurtman, R. J. (1974). Control of brain serotonin levels by the diet. Advances in Biochemical Psychopharmacology, 11, 133-142.

Garelis, E., Young, S. N., Lal, S., and Sourkes, T. L. (1974). Monoamine metabolites in lumbar CSF: the question of their origin in relation to clinical studies. Brain Research, 79, 1-8.

Hochwald, G. M., Wald, A., DiMattio, J., and Malhan, C. (1974). The effects of serum osmolarity on cerebrospinal fluid volume flow. Life Sciences, 15, 1309-1316.

Madras, B. K., Cohen, E. L., Fernstrom, J. D., Larin, F., Munro, H. N., and Wurtman, R. J. (1973). Dietary carbohydrate increases brain tryptophan and decreases free plasma tryptophan. Nature, 244, 34-35.

Modigh, K. (1975). The relationship between the concentrations of tryptophan and 5-hydroxyindoleacetic acid in rat brain and cerebrospinal fluid. Journal of Neurochemistry, 25, 351-352.

Moir, A. T. B., Ashcroft, G. W., Crawford, T. B. B., Eccleston, D., and Guldberg, H. C. (1970). Cerebral metabolites in cerebrospinal fluid as a biochemical approach to the brain. Brain, 93, 357-368. 
Perez-Cruet, J., Chase, T. N., and Murphy, D. L. (1974). Dietary regulation of brain tryptophan metabolism by plasma ratio of free tryptophan and neutral amino acids in humans. Nature, 248, 693-695.

Perez-Cruet, J., Tagliamonte, A., Tagliamonte, P., and Gessa, G. L. (1971). Stimulation of serotonin synthesis by lithium. Journal of Pharmacology and Experimental Therapeutics, 178, 325-330.

Tagliamonte, A., Tagliamonte, P., Perez-Cruet, J., and Gessa, G. L. (1971). Increase of brain tryptophan caused by drugs which stimulate serotonin synthesis. Nature New Biology, 229, 125-126.

Young, S. N., Garelis, E., Lal, S., Martin, J. B., MolinaNegro, P., Ethier, R., and Sourkes, T. L. (1974). Tryptophan and 5-hydroxyindoleacetic acid in human cerebrospinal fluid. Journal of Neurochemistry, 22, 777-779.
Young, S. N., Lal, S., Feldmuller, F., Sourkes, T. L., Ford, R. M., Kiely, M., and Martin, J. B. (1976). Parallel variation of ventricular CSF tryptophan and free serum tryptophan in man. Journal of Neurology, Neurosurgery, and Psychiatry. (In press.)

Young, S. N., Lal, S., Martin, J. B., Ford, R. M., and Sourkes, T. L. (1973). 5-Hydroxyindoleacetic acid, homovanillic acid and tryptophan levels in CSF above and below a complete block of CSF flow. Psychiatria, Neurologia et Neurochirurgia, 76, 439-444.

Young, S. N., Lal, S., Sourkes, T. L., Feldmuller, F., Aronoff, A., and Martin, J. B. (1975). Relationships between tryptophan in serum and CSF and 5-hydroxyindoleacetic acid in CSF of man: effect of cirrhosis of liver and probenecid administration. Journal of Neurology, Neurosurgery, and Psychiatry, 38, 322-330. 